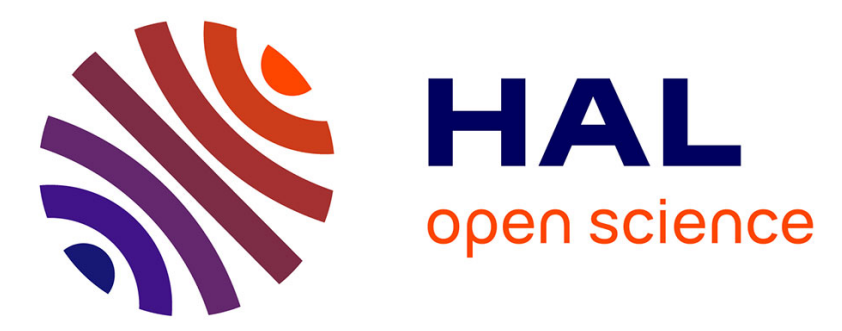

\title{
Sub-adult aging method selection (SAMS): A decisional tool for selecting and evaluating sub-adult age estimation methods based on standardized methodological parameters
}

Louise Corron, François Marchal, Silvana Condemi, Pascal Adalian, David Navega

\section{To cite this version:}

Louise Corron, François Marchal, Silvana Condemi, Pascal Adalian, David Navega. Sub-adult aging method selection (SAMS): A decisional tool for selecting and evaluating sub-adult age estimation methods based on standardized methodological parameters. Forensic Science International, 2019, 304, pp.109897. 10.1016/j.forsciint.2019.109897 . hal-02383161

\section{HAL Id: hal-02383161 \\ https://hal.science/hal-02383161}

Submitted on 15 Dec 2020

HAL is a multi-disciplinary open access archive for the deposit and dissemination of scientific research documents, whether they are published or not. The documents may come from teaching and research institutions in France or abroad, or from public or private research centers.
L'archive ouverte pluridisciplinaire HAL, est destinée au dépôt et à la diffusion de documents scientifiques de niveau recherche, publiés ou non, émanant des établissements d'enseignement et de recherche français ou étrangers, des laboratoires publics ou privés. 


\section{Sub-adult Aging Method Selection (SAMS): a decisional tool for selecting and evaluating sub-adult age estimation methods based on standardized methodological parameters}

Louise Corron, $\mathrm{PhD}^{\mathrm{a}, \mathrm{b}^{*}}$, Pascal Adalian, $\mathrm{PhD}^{\mathrm{a}}$, Silvana Condemi, $\mathrm{PhD}^{\mathrm{a}}$, François Marchal, $\mathrm{PhD}^{\mathrm{a}}$, David Navega, $\mathrm{MSc}^{\mathrm{C}}$

aUMR 7268 ADES - Aix-Marseille University - EFS - CNRS, Faculté de Médecine de Marseille Secteur Nord 51, CS80011, Boulevard Pierre Dramard 13344 Marseille cedex 15, France ${ }^{b}$ Department of Anthropology, University of Nevada, Reno

'Laboratory of Forensic Anthropology, Department of Life Sciences, University of Coimbra, Portugal

\section{*Corresponding author: Icorron@unr.edu}

Contacts: pascal.adalian@univ-amu.fr, silvana.condemi@univ-amu.fr, francois.marchal@univ-amu.fr, dsnavega@gmail.com

\section{ABSTRACT}

Objectives: A decisional tool was developed to select sub-adult age estimation methods referenced in a centralized database. Through a freely accessible webpage interface, this tool allows users to evaluate how much the sampling and statistical protocols of these referenced methods comply with methodological recommendations published for building and applying methods in forensic anthropology.

Materials and methods: 261 publications on sub-adult age estimation were collected. Three search parameters describing the anatomical element(s) and the indicators used to obtain age estimates are chosen by the user to filter the database and present the publications that best correspond to the user's selection. A simple algorithm was created to score age estimation methods according to their relevance and validity. "Relevance" and "Validity" parameters indicate how much a publication complies with user queries and published methodological recommendations, respectively; "Score" is a combination of "Relevance" and "Validity". The closer these parameters are to 1 , the better the method complies with the user's choice and standardized protocols.

Results: The publications resulting from the user's query appear as search results alphabetically. They are characterized by their "Relevance", "Validity" and "Score" values and descriptors relating to their methodology, sampling and statistical protocols. The reference of the publications and an URL to access them online are also provided.

Conclusions: SAMS is a decisional tool based on a centralized database for selecting, accessing and evaluating sub-adult age estimation methods based on published methodological recommendations. Protocol validity can be easily and fully accessed to provide the necessary information for method evaluation. The database will be gradually updated and implemented as new sub-adult age estimation methods are made available online. 


\section{KEYWORDS}

subadult; age estimation; decisional tool; SAMS; Forensic Anthropology Population Data

\section{INTRODUCTION}

Bioanthropologists, bioarcheologists, forensic practitioners, or radiologists can choose from a variety of methods to estimate the skeletal or dental age of living or deceased juvenile or sub-adult individuals. Even though most of these methods are published in scientific journals and/or well-known osteology or bioanthropology books, different levels of personal, institutional, or even national propensity, on top of the significant number of available methods, result in the routine application of only a few of these methods in anthropological practice (1). As a result, although hundreds of methods are available for subadult age estimation, many remain unknown or less known in the bioanthropological contexts they are primarily applied in, e.g. the forensic and bioarcheological contexts. In addition, the medical community, namely practitioners who monitor children's skeletal and dental growth and development, could also benefit from an expanded dissemination of these methods. Therefore, the need for subadult age estimation methods to be made more readily and easily exceeds the limits of the anthropological realm alone.

The forensic anthropological context now goes hand in hand with the requirement for all methods to comply with scientific standards in a legal setting, such as the Daubert criteria $(2,3)$. The methodological standards that must be met in order to fulfill this requirement were presented in several publications over the past ten years (2-6). They objectively highlight the need for standardized methodological sampling and statistical protocols for anthropological methods, in order for them to be valid and applicable in a forensic context. The authors of these papers provide guidelines to help with valid and standardized method construction: these guidelines concern both the samples and sampling protocols used to build the methods, such as the age, sex, and their distributions, and several statistical parameters, such as reliability, accuracy, or standard error of estimation. All of these parameters should 1 / be clearly presented in all publications and $2 /$ follow these guidelines for methodological validity. Indeed, sampling and statistical parameters can be used to assess the methodological "quality" and the scientific and biological validity of the protocols of sub-adult age estimation methods. Because of the various approaches used to build these methods, the significant amount of publications and the methodological and statistical evolution that occurred since the first subadult age estimation method was published in the early $20^{\text {th }}$ century (1), it comes as no surprise that these methods can be extremely variable. Therefore, the accuracy and reliability of the resulting age estimates are quite variable and not always comparable.

The authors presented these standardized methodological guidelines as being crucial to the scientific validity of methods that would in turn ensure valid results. Therefore, these 
guidelines and the sampling and statistical parameters they deem as valid can be used to objectively evaluate methods and their protocols. A previous study has shown that, according to these parameters, a high number of methods do not comply with all recommendations (1). Standardization can also ensure that the estimates obtained with these methods are objectively evaluated and comparable to one another, using statistical parameters such as accuracy, reliability and standard estimation errors, when available. Methods that do not provide such parameters or do not comply with published recommendations should therefore be used with caution at least, or recalibrated or put aside at best.

Protocol standardization and these methodological guidelines for building and applying methods are the main driver for the present anthropological tool: Sub-adult Aging Method Selection, or SAMS. SAMS is a decisional tool freely accessible through a webpage. It was developed to help anthropologists select the methods best adapted to their scientific goals referenced in a large database and directly and objectively evaluate the quality of these methods, according to the sampling and statistical recommendations presented above. SAMS can be found at the following address: osteomics.com/SAMS.

Instructions on how to use SAMS can be found on the webpage, under the "About" tab.

\section{MATERIAL AND METHODS}

The database and parameters

A database referencing 261 publications available online through full-text archives of biomedical and life sciences journal literature and books used for sub-adult age estimation in bioarcheology and/or forensic anthropology was created. This living database can be updated with new publications or with missing information of already referenced methods. It was assembled and used for a study evaluating the validity of sampling and statistical protocols of sub-adult age estimation methods in forensic anthropology $(1,7)$.

Publications were referenced by first author name and original year of publication (first and second author if they are only two, first author et al. if there are more than two). Each publication recorded in SAMS was characterized by several types of parameters: three relate to the anatomical element and age indicator used for age estimation (Table 1) and 19 parameters relate to their sampling, methodological and statistical parameters (Table 2). The first three parameters are selective: they are the ones used as search parameters to filter the database (see How SAMS works section, below). The 19 other parameters are illustrative and are used to characterize and/or evaluate the methods. The parameters were either explicitly presented in the original publications themselves, or they were presented in other reference works on sub-adults and age estimation that revised or tested the methods. If no information was provided in the original publication or other scientific articles using this publication, the parameter is attributed an "Unknown" descriptor (Table 2). To ensure uniformity and comparability between all methods and prevent bugging during processing, the parameters were standardized and simplified to be as binomial as possible. This ensures accuracy is 
maintained as much as possible while decreasing heterogeneity between categories without sacrificing the accuracy of the categories representing the parameters (see Corron et al. 2018 (1) for a full explanation on the process of building the database).

Table 1 Search parameters input by users to filter the database

\begin{tabular}{|c|c|}
\hline $\begin{array}{c}\text { Search } \\
\text { parameters }\end{array}$ & Definition \\
\hline $\begin{array}{c}\text { Anatomical } \\
\text { Region }\end{array}$ & $\begin{array}{l}\text { This parameter acts as a first filter for identifying the anatomical element(s) } \\
\text { on which the age indicators will be obtained from. Several anatomical } \\
\text { regions can be selected, depending on the number of elements users have } \\
\text { at their disposal. }\end{array}$ \\
\hline Bone & $\begin{array}{l}\text { This parameter is essentially the selection of the skeletal and/or dental } \\
\text { element from which the variables used for age estimation are obtained. It is } \\
\text { either a single bone (e.g. humerus), several bones (e.g. limb bones: } \\
\text { humerus, radius, ulna, femur, tibia and fibula), an anatomical zone (e.g. } \\
\text { hand/wrist), or specific developmental states (e.g. deciduous teeth, mixed } \\
\text { dentition, permanent teeth). First, the user selects the general anatomical } \\
\text { region of interest which narrows the choices to the corresponding skeletal } \\
\text { or dental elements of the selected region. Several elements can be selected } \\
\text { for search. }\end{array}$ \\
\hline $\begin{array}{c}\text { Indicator } \\
\text { Type }\end{array}$ & $\begin{array}{l}\text { Indicator type refers to the predictor variables: they are either quantitative } \\
\text { data, such as skeletal measurements, or qualitative data, such as skeletal or } \\
\text { dental development or skeletal maturation stages. Depending on skeletal or } \\
\text { dental preservation, the user can sometimes only use one type of indicator } \\
\text { to predict age and methods are often based on one type or the other. }\end{array}$ \\
\hline
\end{tabular}

\section{How SAMS works}

The idea of SAMS was to build a "smart" classification of the methods in the database, that would a: comply with a user's choice of parameters; and b: comply with published methodological recommendations.

A SAMS user starts by selecting features for the three search parameters: "Anatomical Region", "Bone", "Indicator Type" to filter the database. Several features can be selected for each parameter (Figure 1). The user can also choose all, some, or none of the descriptive parameters to appear in the search results. 
Table 2 Summary of the descriptive parameters of the publications and their respective features appearing in the results filtered by SAMS, as defined for the evaluation of the methods. Bolded features are considered valid according to methodological recommendations

\begin{tabular}{|c|c|c|}
\hline $\begin{array}{l}\text { Type of } \\
\text { parameter }\end{array}$ & Parameter & Features \\
\hline \multirow{7}{*}{ Sampling } & Sample size & $\begin{array}{l}\text { Sample size was arbitrarily subdivided into five categories from } \\
\text { less than a hundred individuals to more than a thousand: }[<100] \text {, } \\
{[100-200],[200-500],[500-1000],[>1000] \text {. }}\end{array}$ \\
\hline & Sample origin & $\begin{array}{l}\text { The geographical origin of the individuals in the sample used to } \\
\text { build the method, mainly relating to the country of origin of the } \\
\text { individuals, but sometimes corresponding to an "ethnic" group or } \\
\text { sub-population affiliation. The methods referenced in SAMS cover } \\
29 \text { countries so far. }\end{array}$ \\
\hline & $\begin{array}{c}\text { Sample } \\
\text { chronology }\end{array}$ & $\begin{array}{l}\text { This parameter informs the user on the general chronological } \\
\text { period the sample used to build the method dates from. The } \\
\text { periods included are contemporary (the sample dates from the } \\
\text { same time the method was published), modern ( } 18^{\text {th }} \text { and } 19^{\text {th }} \\
\text { centuries), and archeological ( } 17^{\text {th }} \text { century and earlier). }\end{array}$ \\
\hline & Study Type & $\begin{array}{l}\text { The type of study was either cross-sectional, semi-longitudinal or } \\
\text { longitudinal. A cross-sectional study uses different and random } \\
\text { individuals, who only contribute once to the sample, at a given } \\
\text { point in time, by the developmental state they are in at that } \\
\text { moment. One individual belongs to one age group. A longitudinal } \\
\text { study follows the same individuals from a starting point to a finish } \\
\text { point in time, and data is repeatedly collected at specific moments } \\
\text { for each individual between these two dates. }\end{array}$ \\
\hline & Age Range & $\begin{array}{l}\text { The precise age ranges of the individuals from the study sample on } \\
\text { which the method was built. This parameter varies greatly from } \\
\text { method to method, covering different ranges of ages from birth to } \\
\text { adulthood. This gives an indication on whether the method is } \\
\text { relevant to the user or not if the bone(s) available for estimation } \\
\text { correspond to the methodological age range or not. }\end{array}$ \\
\hline & Age & $\begin{array}{l}\text { - Known: chronological age is known for all individuals in the study } \\
\text { sample } \\
\text { - Unknown: chronological age is not known for any individual in } \\
\text { the study sample. It is often estimated beforehand using a } \\
\text { different method } \\
\text { - Known and unknown: chronological age is known for some } \\
\text { individuals in the study sample }\end{array}$ \\
\hline & Sex & $\begin{array}{l}\text { - Known: sex is known for all individuals in the study sample } \\
\text { - Unknown: sex is not known for any individual in the study sample } \\
\text { - Known and unknown: sex is known for some individuals in the } \\
\text { study sample }\end{array}$ \\
\hline
\end{tabular}




\begin{tabular}{|c|c|c|}
\hline & $\begin{array}{l}\text { Uniformity of } \\
\text { age } \\
\text { distribution }\end{array}$ & $\begin{array}{l}\text { - Yes: the distribution of the number of individuals per age group } \\
\text { (monthly, annual) in the study sample is even } \\
\text { - No: the distribution of the number of individuals per age group } \\
\text { (monthly, annual) in the study sample is not even } \\
\text { - Unknown: the distribution of the number of individuals per age } \\
\text { group (monthly, annual) in the study sample is unknown }\end{array}$ \\
\hline & $\begin{array}{l}\text { Uniformity of } \\
\text { sex } \\
\text { distribution }\end{array}$ & $\begin{array}{l}\text { - Yes: the distribution of the number of individuals per age group } \\
\text { (monthly, annual) in the study sample is even for both sexes } \\
\text { - No: the distribution of the number of individuals per age group } \\
\text { (monthly, annual) in the study sample for both sexes is not even } \\
\text { - Unknown: the distribution of the number of individuals per age } \\
\text { group (monthly, annual) in the study sample for both sexes is } \\
\text { unknown }\end{array}$ \\
\hline \multirow{7}{*}{ Statistical } & $\begin{array}{l}\text { Reliability of } \\
\text { estimated age }\end{array}$ & $\begin{array}{l}\text { - } \geq 95 \% \text { : reliability of estimated age is equal to or higher than } 95 \% \\
\text { - < 95\%: reliability of estimated age is lower than } 95 \% \\
\text { - Unknown: reliability of estimated age is unknown }\end{array}$ \\
\hline & $\begin{array}{l}\text { Accuracy of } \\
\text { estimated age }\end{array}$ & $\begin{array}{l}\text { - Known: accuracy of estimated age is known } \\
\text { - Unknown: accuracy of estimated age is unknown }\end{array}$ \\
\hline & $\begin{array}{l}\text { Detailed } \\
\text { accuracy }\end{array}$ & $\begin{array}{l}\text { The exact accuracy value of the age estimates of the } \\
\text { corresponding method, when known. }\end{array}$ \\
\hline & $\begin{array}{l}\text { Standard } \\
\text { error of } \\
\text { estimation }\end{array}$ & $\begin{array}{l}\text { - Known: standard error of estimation or the size of the prediction } \\
\text { interval of estimated age is known } \\
\text { - Unknown: standard error of estimation or the size of the } \\
\text { prediction interval of estimated age is unknown }\end{array}$ \\
\hline & Detailed SEE & $\begin{array}{l}\text { The exact value of the standard estimation error of age of the } \\
\text { corresponding method, when known. }\end{array}$ \\
\hline & $\begin{array}{l}\text { Repeatability } \\
\quad \text { and } \\
\text { reproducibility }\end{array}$ & $\begin{array}{l}\text { - Sufficient repeatability and reproducibility: repeatability and } \\
\text { reproducibility of the age indicators used for age estimation were } \\
\text { both tested beforehand or in a different publication } \\
\text { - Sufficient repeatability: repeatability of the age indicators used } \\
\text { for age estimation was tested beforehand } \\
\text { - Sufficient reproducibility: reproducibility of the age indicators } \\
\text { used for age estimation was tested beforehand or in a different } \\
\text { publication } \\
\text { - Untested repeatability and reproducibility: neither repeatability } \\
\text { or reproducibility of the age indicators used for age estimation } \\
\text { were tested } \\
\text {-Unknown: repeatability and reproducibility is unknown }\end{array}$ \\
\hline & $\begin{array}{l}\text { Validation of } \\
\text { the method }\end{array}$ & $\begin{array}{l}\text { - Validated method or data: the published data or age estimation } \\
\text { method was validated by the authors using cross-validation and/or } \\
\text { an independent test sample and/or was validated by an } \\
\text { independent study } \\
\text { - Non-validated method or data: no published validation of the } \\
\text { method was found in the literature }\end{array}$ \\
\hline Other & Sexed Method & $\begin{array}{l}\text { Whether the method was built separately for males and females or } \\
\text { not. } \\
\text { Two features: Yes or No }\end{array}$ \\
\hline
\end{tabular}




\begin{tabular}{|c|c|}
\hline Variable Type & $\begin{array}{l}\text { The specific variable type used for age prediction (e.g. bone } \\
\text { measurement, dental mineralization stage, etc.) in the } \\
\text { corresponding method. This parameter is extremely variable, and } \\
\text { we advise the user to verify the methods' applicability in the original } \\
\text { publication depending on the preservation of the elements } \\
\text { available. }\end{array}$ \\
\hline $\begin{array}{c}\text { Medium of } \\
\text { Study }\end{array}$ & $\begin{array}{l}\text { The medium used for acquisition of the predictor variables in the } \\
\text { corresponding method, such as dry bone, radiography, computed } \\
\text { tomography, biochemical analysis, etc. }\end{array}$ \\
\hline Result Type & $\begin{array}{l}\text { This parameter is a general description of how the method is } \\
\text { applied and how the results are obtained. It provides additional } \\
\text { information on the method for the user, prior to its application. } \\
\text { Result types were assigned into } 6 \text { categories: } \\
\text { - based on age per stage/score, } \\
\text { - based on descriptive statistical parameters (mean, sd, } \\
\text { frequencies, etc.), } \\
\text { - based on regression equations, } \\
\text { - based on atlas, abacus, tables, diagrams or growth curves, } \\
\text { - based on probabilistic inference, } \\
\text { - unknown. }\end{array}$ \\
\hline
\end{tabular}

The search parameters are the basis of the classification. The user selects features for the three search parameters relating to the anatomical element(s) at their disposal and the type of age indicator (quantitative, qualitative, both or either) they can obtain from the element(s) for age estimation. Descriptive parameters are different sampling, methodological and statistical characteristics presented in the publications and integrated in the database that provide additional information to help users with method selection and evaluation. The user can choose none or all of these features to appear in the results. After selecting the desired features, the user clicks on the "Filter" button to visualize the results. The database is then filtered following a simple similarity-based algorithm to provide the results. 
Fig. 1. Example of a SAMS query. This query uses several features of the three search parameters to filter the database and part of the descriptive parameters that will appear in the results.

The algorithm behind SAMS

SAMS uses a very simple similarity-based algorithm to compare user's input data to indexed publications and determine their relevance, which in this context means assess if the keywords regarding anatomical region and skeletal elements match those of the indexed methods. Relevance (R) is computed as follows: if the user-selected input matches the publications' keywords for anatomical region and bones involved, a value of 1 is attributed; if not, a value of 0 is given instead. The calculated average of these comparison values gives the relevance value $(R)$ in a normalized range between 0 (not relevant) and 1 (highly relevant) for all 261 methods in the database. The same reasoning is applied to compute the validity value (V) of the methods by assessing the similarity of a given publication to each of the ten validity parameters (see Table 3). The final score (S) is given by the square root of the product of the relevance and validity values $\left(S=V R^{*} V\right)$. Only methods with a relevance higher than zero are retrieved.

\section{RESULTS}

The methods filtered using the features of the three search parameters and the algorithm behind SAMS appear as results on the page, along with any of the descriptive parameters the user has chosen to visualize (Figure 2). A URL provides a link to find the corresponding publications online.

The methods resulting from a query are classified from most to least compliant with the query via their "Relevance" value. The higher the "Relevance" of a method (the closer that value is to one), the closer the method is to the features the user selected for the three search parameters. A relevance of one means all the features of the three search parameters are the same as the features selected by the user. 


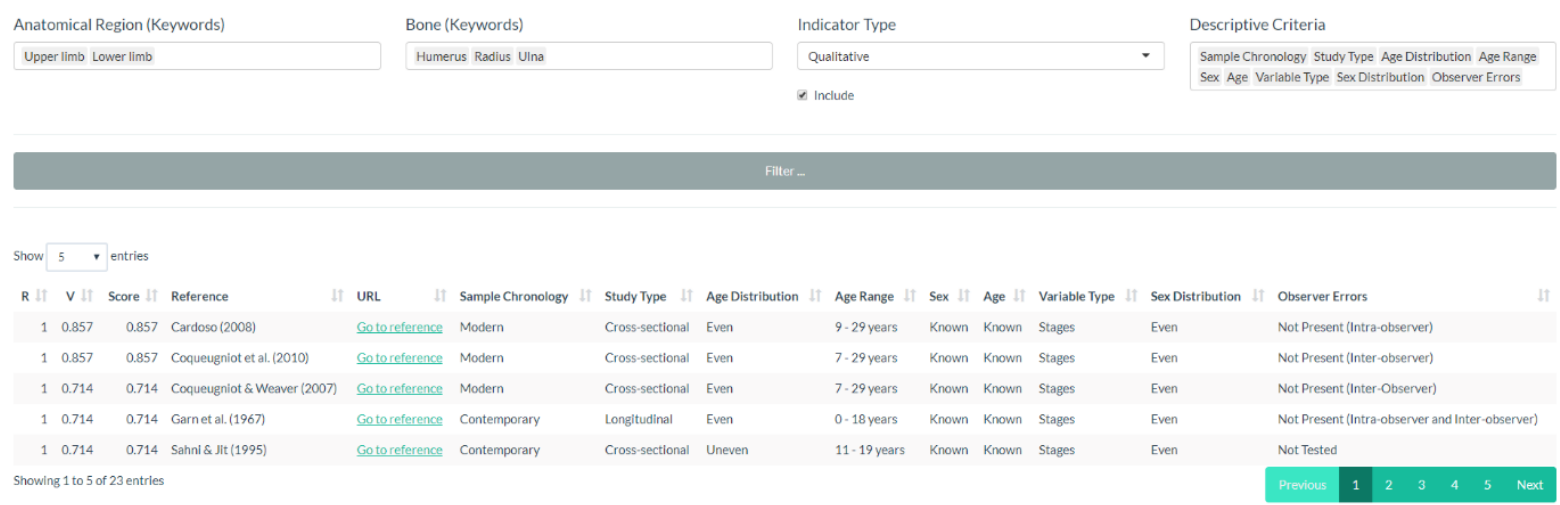

Fig. 2. Results of the query obtained by filtering the database using the SAMS algorithm. The features of the three search parameters are presented at the top. Users can evaluate the methods using the Relevance (R), Validity (V) and Score.

The methods are also classified from best to least compliant with published recommendations via their "Validity" value. The higher the "Validity" value (the closer that value is to one), the closer the methods comply with published sampling and statistical recommendations (see Table 3). A method with a validity value of one means that method complies with all valid features for the four sampling parameters and the five statistical parameters. The four valid sampling features are namely samples comprised of individuals of known age and known sex, with even age and sex distributions across age groups; the five valid statistical features are a reliability equal to or higher than 0.95 , known accuracy, known standard estimation error, sufficiently low intra- and inter-observer errors, and the existence of some form of methodological validation.

Finally, each publication is awarded a "Score" value, which is the multiplication of the "Relevance" and "Validity" values (Figure 2 and Table 3). The higher the "Score" (the closer it is to one), the better the methods comply with both user demands ("Relevance") and methodological recommendations ("Validity").

\section{DISCUSSION}

\section{Building SAMS - a compromise between reliability and precision of the parameters}

The objectivity of the results is based on how the parameters of the methods were "translated" and categorized according to published recommendations. Standardization of these parameters to fit them into mainly binomial categories sometimes required simplification (see Corron et al. 2018, (1)). This is a common issue when dealing with categorical data in science (11). The authors made the choice to privilege reliability of the information by reducing the number of descriptors rather than precision, which would require a higher number of categories to describe the methodological parameters. Indeed, on top of decreasing the reliability of the results, the latter option of keeping a higher number of 
categories would also prevent direct comparison between the same methodological parameters of the methods in the output. This would go against the entire purpose of method classification and evaluation using common standardized parameters. This is why SAMS is presented as a guide to select, compare and evaluate methods. Once one or several methods are selected, the users are invited to visualize and read the selected publication(s) thoroughly in order to familiarize themselves with the details of the method(s) before applying it and including it in the report. URL links to access each method are presented in the output long with the descriptive parameters.

Table 3 Definitions of the result variables used to measure the relevance of the decisional tool and evaluate method compliance with published recommendations on method validity

\begin{tabular}{|c|c|}
\hline Variables & Definition \\
\hline $\begin{array}{c}R: \\
\text { Relevance }\end{array}$ & $\begin{array}{l}\text { Relevance corresponds to how much the methods are close to the } \\
\text { features selected for the three search parameters selected: Anatomical } \\
\text { region, Bone and Indicator type. The higher the Relevance (i.e. the closer } \\
\text { it is to 1), the closer the method is to the user's selected search } \\
\text { parameters. }\end{array}$ \\
\hline V: Validity & $\begin{array}{l}\text { Validity is how much the methods respect what are considered valid } \\
\text { sampling and statistical parameters by the authors of methodological } \\
\text { guidelines cited previously. } \\
\text { In this context of juvenile age estimation, the valid parameters are the } \\
\text { following: } \\
\text { - } \quad 4 \text { sampling parameters: samples with individuals of known age } \\
\text { and known sex, even age and sex distributions across age groups. } \\
\text { - } 5 \text { statistical parameters: reliability }=0.95+\text {, known accuracy, } \\
\text { known SEE, sufficiently low intra- and inter-observer errors, and } \\
\text { the existence of some form of methodological validation. } \\
\text { Publications with high V values (i.e. closer to } 1 \text { ) comply the most with } \\
\text { these methodological guidelines. }\end{array}$ \\
\hline S: Score & $\begin{array}{l}\text { Score }=\text { Relevance }{ }^{*} \text { Validity. This composite value gives an overall } \\
\text { ranking of the publications according to } \mathrm{R} \text { and } \mathrm{V} \text { values. Publications } \\
\text { with high } \mathrm{S} \text { values are the ones that correspond to the users' choice AND } \\
\text { respect methodological guidelines the most. }\end{array}$ \\
\hline
\end{tabular}

Some methods, mainly older publications, presented incomplete or unclear sampling and/or statistical parameters were concerned. To account for this, the "Unknown" category was added to the selective parameters (see Corron et al. 2018, (1)). This can potentially modify the outcoming validity and score values if the unknown categories concern one or several of the nine parameters for which validity or invalidity was assessed using methodological recommendations (3-6). If this information is incorrect, it can be easily fixed 
by the authors who can modify the dataset as soon as the correct information is made available to them and verified. Another limitation is the restricted access to publications. This is either because publications have not been digitized or are not available online, especially for older methods, or because of the language barrier, preventing the authors from including works originally published in languages other than English or French. If additional publications are made available online or directly sent to the authors, they could easily be integrated to the database and increase the number of references. SAMS can therefore stay relevant and improve with time through the collaborative effort of anthropologists who aim to standardize methods and share knowledge around the world.

With the wide array of methods or approaches available to anthropologists for estimating subadult age comes some level of variation in terms of sampling and statistical parameters and statistical approaches. If some of these parameters, such as the nine ones we selected as classifying parameter for SAMS, can effectively be categorized as valid or invalid based on published recommendations, it is more difficult to so confidently assign a valid or invalid stamp for the type of statistical approach used to estimate age. In our sample of 261 publications, the "Result type" parameter was reduced to five categories (Table 2) which themselves each cover between one and five different statistical approaches.

Although some publications have looked into determining which statistical approach is the "best" for age estimation using ordinal or continuous data $(1,8-10)$, they do not provide a consensual or clear answer that is applicable for all indicators of age, and/or all ages and/or all contexts (e.g. forensic or bioarcheological). This does not seem surprising based on the variety of data these methods are based on. The different levels of variation behind the age indicators themselves also probably play a role in these diversified approaches. Because of these limitations, we chose not to classify "Results type" as a classification parameter for method evaluation. However, because of its unequivocal value for method selection, we decided to keep it as a descriptive parameter in SAMS that the user could choose to use as a selective parameter if desired. As more papers are published on subadult age estimation, this parameter, and others, could ultimately be included as classifying parameters to improve the accuracy of the relevance and validity values to better qualify the methods.

\section{Using SAMS - Applications and limitations}

The main goal of SAMS is to provide anthropologists with a practical analytical tool that follows recommendations for building and applying subadult age estimation methods in the forensic context in order to assist practitioners. This type of approach could of course be adapted to any type of method, be it adult age estimation, sex estimation, or other, and could be based on other recommendations or protocols. Presenting SAMS as a free tool available online facilitates the dissemination of all the methods included in this database to all bioanthropological and medical contexts. To our knowledge, there is no tool comparable to SAMS available in the forensic anthropology or larger bioanthropological community. Several GUIs have been developed these past few years to facilitate practical method application and dissemination, but they are all method-specific and are not a meta-analytical decisional tool. 
By helping decipher and evaluate published methods based on common standardized parameters, SAMS also helps clearly present the advantages and limits of each method appearing as a query result. These advantages and limits are elements that should be included in technical reports, especially in the forensic context. By dissecting each element of a method's sampling and statistical protocols, SAMS provides direct criticism of that method to help understand and evaluate the methodology behind the publication for more objective and critical report writing. A better understanding of the method by the practitioner will improve the clarity of the report and a better understanding of the methods by the judicial figure reading the report. By allowing for protocol transparency and standardization, SAMS enables both the practitioner and the judge to easily evaluate whether a method complies with the Daubert criteria or not. Indeed, SAMS acts as a screening process allowing each methodological parameter to be deciphered and separated, so the defaulting or limiting parameter(s) for each method can be easily spotted. The relevance and validity values emphasize this evaluation by objectively providing statistical arguments to the confidence one can have in a method's quality, i.e. how much it complies with the Daubert recommendations.

Although SAMS is based on the methodological rigor necessary in the forensic context, it is also useful in the archeological context. Its main appeal lies in the wide range of methods included in it that can be selected. Indeed, the main issue in bioarcheology is the variation in skeletal preservation (12). As a database compiling methods for almost every anatomical element of the skeleton, SAMS increases the chances of finding one or several methods compatible with the available remains. Moreover, it provides a better visibility for methods that are sometimes forgotten or not well-disseminated (e.g. methods published in regional/country-specific journals, older methods unavailable online). Overall, SAMS helps broaden the methodological possibilities of subadult age estimation, which is beneficial for the study of subadult individuals in bioarcheological, forensic or pediatric contexts.

\section{CONCLUSIONS}

SAMS provides a quick and easy way to help forensic anthropology practitioners and bioarcheologists select and evaluate an ensemble of sub-adult age estimation methods. It provides relevant information related to sampling and statistical parameters of these methods to verify whether they comply with published recommendations on anthropological methods in a forensic setting through their "Validity" scores.

SAMS will continue to be implemented with new publications to ensure it stays relevant and up to date as more and more sub-adult age estimation methods are published. $\mathrm{Bi}$-annual updates are planned to input new relevant publications.

As we aim to adopt a collaborative approach, we welcome any scientific support the anthropology community can make to complete, correct and update this work. 


\section{CRediT authorship contribution statement}

Louise Corron: Conceptualization, Methodology, Data curation, Writing - original draft, Writing - review \& editing, Software, Visualization. Pascal Adalian: Supervision, Validation, Writing - review \& editing, Resources, Project administration, Funding acquisition. Silvana Condemi: Supervision, Validation, Project administration, Funding acquisition. François Marchal: Supervision, Validation, Writing - review \& editing, Resources, Project administration, Funding acquisition. David Navega: Software, Methodology, Data curation, Formal analysis, Investigation, Visualization.

\section{Acknowledgments}

The authors wish to thank Prof Eugenia Cunha and Dr Kyra Stull for their help and advice on how to build SAMS and Ms Laura Cirillo, Dr Aurore Lambert, Dr Sandy Parmentier, Dr Emeline Verna and Ms Tatiana Vlemincq-Mendieta for their feedback.

\section{REFERENCES}

1. Corron L. Marchal F. Condemi S. Adalian P. 2018 A critical review of sub-adult age estimation in biological anthropology: do methods comply with published recommendations? Forensic Science International 288:328.e1-328.e9

2. Cattaneo C. 2007 Forensic anthropology: Developments of a classical discipline in the new millenium. Forensic Science International 165:185-193

3. Cunha E. Baccino E. Martrille L. Ramsthaler F. Prieto J. Schuliar Y. Lynnerup N. Cattaneo C. 2009 The problem of aging human remains and living individuals: a review. Forensic Science International 193:1-13

4. Ritz-Timme S. Cattaneo C. Collins M.J. Waite E.R. Schütz H.W. Kaatsch H.-J. Borrman H.I.M. 2000 Age estimation: the state of the art in relation to the specific demands of forensic practice. International Journal of Legal Medicine 113:129-136

5. Rösing F.W. Graw M. Marre B. Ritz-Timme S. Rothschild M.A. Rotzscher K. Schmeling A. Schroder I. Geserick G. 2007 Recommendations for the forensic diagnosis of sex and age from skeletons. Homo-Journal of Comparative Human Biology 58(1):75-89

6. Schmeling A. Geserick G. Reisinger W. Olze A. 2007 Age estimation. Forensic Science International 165:178-181

7. Corron L. 2016 Juvenile age estimation in physical anthropology: a critical review of existing methods and the application of two standardised methodological approaches. PhD thesis, Aix-Marseille University, Marseille, 560p. 
8. Konigsberg L.W. Hermann N.P. Wescott D.J. Kimmerle E.H. 2008 Estimation and evidence in forensic anthropology: age-at-death. Forensic Science International 53(3):541-557

9. Konigsberg L.W. Frankenberg S.R. Liversidge H.M. 2016 Optimal trait scoring for age estimation. American Journal of Physical Anthropology 159(4):557-576

10. Nayyar A.S. Babu B.A. Krishnaveni B. Devi M.V. Gayitri H.C. 2016 Age estimation: current state and research challenges. Journal of Medical Sciences 36(6):209-216

11. Powers DA, Xie Y. 2000 Statistical methods for categorical data analysis. San Diego: Academic Press

12. Gordon C.C. Buikstra J.E. 1981 Soil pH, Bone Preservation, and Sampling Bias at Mortuary Sites. American Antiquity 46(3):566-571 